\title{
Fusarium Virulence Assay on Wheat and Barley Seedlings
}

Lorenzo Covarelli ${ }^{\star}$, Donald Gardiner ${ }^{2}$, Giovanni Beccari ${ }^{2}$ and Paul Nicholson ${ }^{3}$

${ }^{1}$ Department of Agricultural and Environmental Sciences, Faculty of Agriculture, University of Perugia, Perugia, Italy; ${ }^{2}$ Division of Plant Industry, CSIRO, Brisbane, Australia; ${ }^{3}$ Department of Crop Genetics, John Innes Centre, Norwich, UK

*For correspondence: lorenzo.covarelli@unipg.it

[Abstract] Fusarium root and crown rot is a very important complex disease of small grain cereals worldwide which may lead to very high yield losses. Traditional virulence assays are time consuming and often require plants to be grown in greenhouses or climatic chambers in soil. We describe a rapid laboratory assay for assessing such a disease in wheat and barley seedlings. The method could be successfully used for testing fungal virulence as well as to assess plant resistance.

\section{Materials and Reagents}

1. Fungal strains

2. $25 \%$ Campbell's V8 juice (pH 6.5 with $\mathrm{NaOH})$

3. $1.2 \%$ agar plates ( $9 \mathrm{~cm}$ diameter)

4. Wheat and barley seeds

5. Sterilizing solution ( $0.64 \%$ sodium hypochlorite $-10 \%$ ethanol)

6. Sterile deionized water

\section{Equipment}

1. Petri dishes (9 and $14 \mathrm{~cm}$ diameter)

2. Filter paper (Whatman No.3, 8 and $12.5 \mathrm{~cm}$ diameter)

3. Pipette tip or cork borer

4. Sealing film (Phyto Technology Laboratories) or parafilm

5. Ruler

6. Razor blades

7. Laminar flowhood

8. Illuminated incubator at $21 \pm 1^{\circ} \mathrm{C}$, light intensity $>90 \mu$ mols

9. Fungal growth cabinet with white (one $18 \mathrm{~W}$ lamp) and black fluorescent (one $18 \mathrm{~W}$ lamp) lights on $12 / 12 \mathrm{~h}$ day/night cycle at $20-22{ }^{\circ} \mathrm{C}$ 
10. Incubator

\section{Procedure}

1. Day 1

Grow fungal colonies on V8 agar plates for 7 days at $20-22{ }^{\circ} \mathrm{C}$ under white and black fluorescent light on a 12/12 $\mathrm{h}$ day/night cycle, starting from stored fungal cultures or colonies from plates.

2. Day 3 (wheat) or 4 (barley)

For each fungal isolate or plant genotype 16 germinated seeds are required. If testing fungal isolate virulence, a mock treatment is also required. Barley is more vigorous in the early stages of growth and seeds are allowed to germinate for 2 days whereas wheat is allowed to germinate for 3 days prior to inoculation.

a. Disinfect wheat or barley seeds in sterilizing solution for 5 min and wash 3 times with sterile deionized water.

b. Imbibe disinfected seeds in $9 \mathrm{~cm}$ Petri dishes containing one $8 \mathrm{~cm}$ filter paper soaked with sterile deionized water at $4{ }^{\circ} \mathrm{C}$ for 1 day. Keep in dark by wrapping plates in aluminium foil.

3. Day 4 (wheat) or 5 (barley)

Transfer plates to plant growth chamber but maintain in darkness (wrapped in foil).

4. Day 6

a. Place three $12.5 \mathrm{~cm}$ diameter filter paper layers into $14 \mathrm{~cm}$ Petri dishes.

b. Add sterile deionized water to filter paper until completely soaked.

c. Remove excess water from plates.

d. Place 16 pre-germinated wheat or barley seeds on filter paper in rows of 3-5-5-3 seeds.

e. Seal plates with film and place plates to plant growth chamber in dark overnight.

5. Day 7

a. Make mycelial plugs of approximately 5-6 mm diameter by using the reverse of a pipette tip or a cork borer from the edge of the growing colony (Figure 1). 


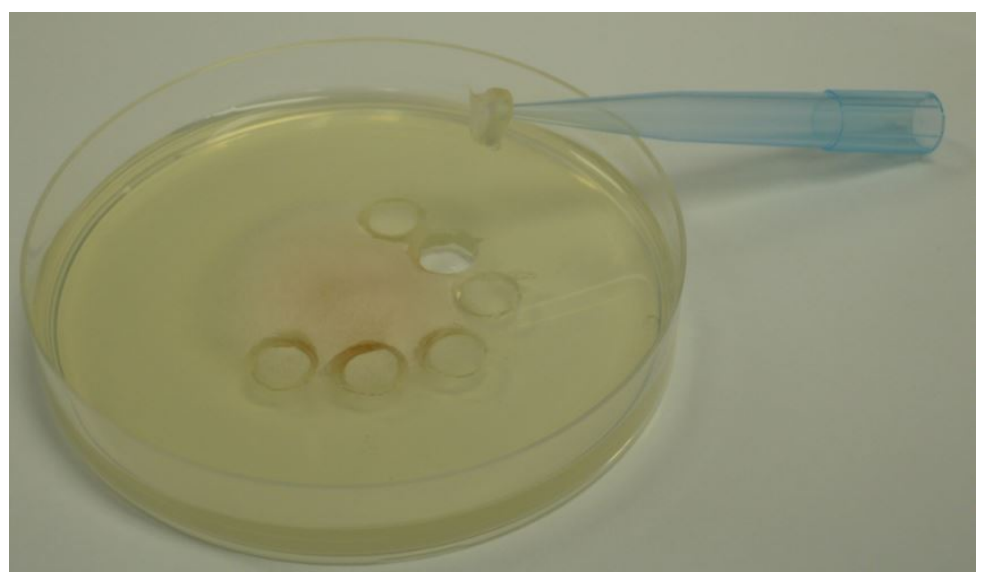

Figure 1. Mycelial plugs obtained from the edge of a growing Fusarium colony.

b. Place mycelial plugs upside down on the main root of each germinated seed when about $3 \mathrm{~cm}$ long, at approximately $1 \mathrm{~cm}$ from the seed, with the mycelium in direct contact with the root (Figure 2).

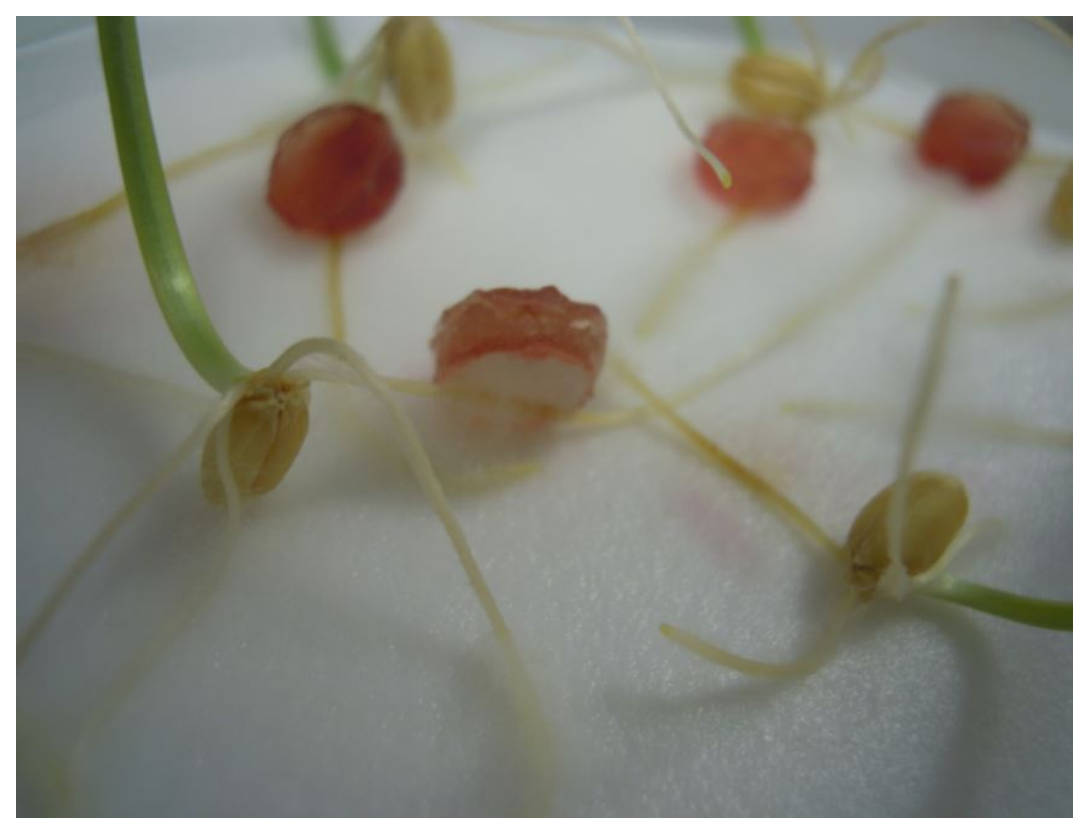

Figure 2. Mycelial plugs on a wheat rootle.

C. Seal plates with sealing film or Parafilm.

d. Put plates in incubator at $21 \pm 1^{\circ} \mathrm{C}$ for 4 to 6 days with $12 \mathrm{~h}$ of light.

6. Day $11-13$ as required

a. Determination of root-rot: observation of root necrosis at 4 days post-inoculation by measuring symptom extension (SE) (cm) and visually observing the browning index 
(BI, $0=$ symptomless; 1 = slightly necrotic; 2 moderately necrotic; 3 = severely necrotic; 4 = completely necrotic).

b. Determination of crown-rot: Visual observation of crown necrosis at 6 days postinoculation by a $0-4$ scale $(0=$ symptomless; 1 = slightly necrotic; 2 moderately necrotic; 3 = severely necrotic; 4 = completely necrotic), measuring of shoot length with a ruler $(\mathrm{cm})$ and weighing the shoots after their dissection with a razor blade.

Please see Figure 1 of Beccari et al. (2011) and Figures 6 and 8 of Gardiner et al. (2012).

\section{Acknowledgments}

The present protocol here described in detail was adopted in experiments reported in the following publications: Beccari et al. (2011) and Gardiner et al. (2012). Work was supported by the Australian Grains Research and Development Corporation, an Australian statutory authority.

\section{References}

1. Beccari, G., Covarelli, L. and Nicholson, P. (2011). Infection processes and soft wheat response to root rot and crown rot caused by Fusarium culmorum. Plant Pathol 60(4): 671-684.

2. Gardiner, D. M., McDonald, M. C., Covarelli, L., Solomon, P. S., Rusu, A. G., Marshall, M., Kazan, K., Chakraborty, S., McDonald, B. A. and Manners, J. M. (2012). Comparative pathogenomics reveals horizontally acquired novel virulence genes in fungi infecting cereal hosts. PLoS Pathog 8(9): e1002952. 